\title{
Novel crosstalk between KLF4 and ZEB1 regulates gemcitabine resistance in pancreatic ductal adenocarcinoma
}

\author{
ZHIYI WANG ${ }^{1}$, YUAN CHEN ${ }^{2}$, YANLIANG LIN $^{3}$, XINXING WANG $^{1}$, XIANPING CUI $^{1}$, \\ ZHENHAI ZHANG $^{1}$, GUOZHE XIAN ${ }^{1}$ and CHENGKUN QIN ${ }^{1}$ \\ Departments of ${ }^{1}$ Hepatobiliary Surgery, ${ }^{2}$ Paediatrics and ${ }^{3}$ Center Laboratory, \\ Shandong Provincial Hospital Affiliated to Shandong University, Jinan, Shandong 250021, P.R. China
}

Received May 26, 2017; Accepted August 8, 2017

DOI: 10.3892/ijo.2017.4099

\begin{abstract}
Pancreatic ductal adenocarcinoma (PDAC) is one of the most lethal malignancies with broad resistance to chemotherapeutic drugs. Krüppel-like factor 4 (KLF4) is a candidate tumor suppressor in PDAC. However, the precise role of KLF4 in gemcitabine resistance of PDAC remains largely unclear. In this study, we demonstrated that gemcitabine inhibited KLF4 expression. Moreover, gemcitabine also reduced the levels of miR-200b and miR-183, but promoted ZEB1 expression in PDAC cells. KLF4 knockdown blocked the expression of miR-200b and miR-183, and inversely, KLF4 overexpression promoted the expression of miR-200b and miR-183, suggesting that KLF4 positively regulated the expression of miR-200b and miR-183. Moreover, KLF4 knockdown enhanced ZEB1 expression and gemcitabine resistance while KLF4 overexpression induced the opposite effect. ChIP assays verified that KLF4 positively regulated the expression of miR-200b and miR-183 by directly binding to their promoters. Then, miR-200b and miR-183 directly inhibited ZEB1 expression by targeting its 3'UTR region. ZEB1 knockdown attenuated gemcitabine resistance in PDAC cells. KLF4 overexpression promoted gemcitabine sensitivity of PDAC in vivo by negatively regulating ZEB1 expression. Our results revealed that novel crosstalk between KLF4 and ZEB1 regulated gemcitabine resistance in PDAC.
\end{abstract}

\section{Introduction}

Pancreatic ductal adenocarcinoma (PDAC) is a highly lethal malignancy with an estimated 5-year survival rate $<5 \%$, and by 2020 , it is estimated to be the second leading cause of

Correspondence to: Dr Guozhe Xian or Professor Chengkun Qin, Department of Hepatobiliary Surgery, Shandong Provincial Hospital Affiliated to Shandong University, Jinan, Shandong 250021, P.R. China

E-mail: xianguozhe@aliyun.com

E-mail: qinchengkun@medmail.com.cn

Key words: gemcitabine,KLF4, miR-200b, miR-183,ZEB1, pancreatic ductal adenocarcinoma cancer-related mortality (1). Due to the lack of early detection methods and effective preventive strategies, PDAC is usually diagnosed at an advanced stage when it is deemed unresectable and exhibits poor response to chemotherapeutic drugs, including gemcitabine and 5-fluorouracil (2). Thus, it is essential to understand the underlying mechanisms for the chemoresistance of PDAC.

Krüppel-like factor 4 (KLF4) is a transcription factor that serves as an important regulator in cell differentiation, cell cycle, cell survival and apoptosis (3). KLF4 exerts seemingly paradoxical functions in different cell types. KLF4 is downregulated in bladder and gastric cancer cells, and enforced expression of KLF4 promotes apoptosis in these cells (4). In PDAC, KLF4 also functions as a tumor-suppressor (5). Conversely, KLF4 exhibits oncogenic functions in oral, skin squamous carcinoma cells and breast ductal carcinoma (6). To date, little is known about the precise role of KLF4 in gemcitabine resistance of PDAC.

The epithelial-mesenchymal transition (EMT)-activator ZEB1 is a transcriptional repressor of epithelial genes, such as E-cadherin (7). ZEB1 has been widely implicated in the invasiveness and metastasis of solid tumors, including lung adenocarcinoma, colorectal and breast cancers (8-10). Ectopic expression of ZEB1 also promotes drug-resistant phenotypes (7). However, the role of ZEB1 in gemcitabine resistance of PDAC remains unexplored.

In this study, we showed that gemcitabine treatment inhibited KLF4 expression and promoted ZEB1 expression. Further investigation demonstrated that KLF4 suppressed ZEB1 expression and gemcitabine resistance by directly regulating the expression of miR-200b and miR-183. In addition, ZEB1 knockdown attenuated gemcitabine resistance in PDAC cells.

\section{Materials and methods}

Antibodies and reagents. The following monoclonal antibodies (mAbs) and polyclonal antibodies (pAbs) were used: anti-KLF4 (pAb,ab106629), and anti-ZEB1 (pAb, ab155249) were purchased from Abcam (Cambridge, MA, USA); anti-GAPDH (pAb) was purchased from Anbo Biotechnology Co. (San Francisco, CA, USA); Cell counting kit- 8 was purchased from Dojindo Laboratories (Tokyo, Japan). Gemcitabine was purchased from Eli Lilly (Indianapolis, IN, USA). ZEB1 siRNA, miR-200b 
and miR-183 were synthesized by Biosune Biotechnology (Shanghai, China).

Cell culture. The human PDAC cell lines BxPC-3, Panc-1 and MIApaca-2 were purchased from the American Type Culture Collection (Manassas, VA, USA). Cells were cultured in RPMI-1640 medium (Hyclone, USA) supplemented with $10 \%$ fetal bovine serum (Hyclone), $100 \mathrm{U} / \mathrm{ml}$ penicillin, $100 \mathrm{U} / \mathrm{ml}$ streptomycin and $0.03 \% \mathrm{~L}$-glutamine at $37^{\circ} \mathrm{C}$ in $5 \% \mathrm{CO}_{2}$.

Cell viability assay. PDAC cells were seeded at a density of $1.0 \times 10^{4}$ cells/well in 96-well microplates with $100 \mu \mathrm{l}$ RPMI-1640 medium. After grown to $\sim 80 \%$ confluence, the cells were incubated with the indicated concentration of gemcitabine. After treatment for $72 \mathrm{~h}$, the cells were incubated with $10 \%$ WST- 8 dye for $2 \mathrm{~h}$ at $37^{\circ} \mathrm{C}$. The absorbance was detected at $450 \mathrm{~nm}$ using a SpectraMax M2. $\mathrm{IC}_{50}$ values were determined using GraphPad Prism 6.0.

Flow cytometric analysis. PDAC cells were seeded at a density of $2 \times 10^{5}$ cells/well in 6 -well microplates. After treatment, flow cytometric analysis was performed to determine cell apoptosis. In brief, after treatment with gemcitabine, the cells were digested using trypsin lacking EDTA and phenol red, and were incubated in binding buffer containing Annexin V-FITC $(2.5 \mathrm{mg} / \mathrm{ml})$ and propidiumiodide $(5 \mathrm{mg} / \mathrm{ml})$ for $10 \mathrm{~min}$ in the dark at room temperature. Subsequently, the labeled cells were detected by a flow cytometer (Beckman Coulter, Chicago, IL, USA).

Lentiviruses construction. For KLF4 knockdown, the short hairpin RNA (shRNA) targeting the coding sequence of KLF4 (TACCCATCCTTCCTGCCCGAT) was digested using the restriction enzymes AgeI and EcoRI (New England Biolabs UK Ltd., UK), and was then cloned into pGCSIL-GFP plasmid. For KLF4 overexpression, the full coding region of KLF4 was amplified using specific primers (forward, 5'-CGCGGATCC GCGATGGCTGTCAGCGACGCG-3'; reverse, 5'-GGGTACC GGTCGCCACCTTCTCTTCTGGCAGTGTGGG-3'). The product was digested using the restriction enzymes BamHI and AgeI, and was then linked to p-GC365-EGFP plasmid. The plasmids were transformed into $E$. coli DH5a. Positive clones were harvested and identified by sequencing. The recombinant plasmids were transfected to $293 \mathrm{~T}$ cells by Lipofectamine 2000 (Invitrogen). The infection efficiency was determined by measuring fluorescence intensity under microscopy (Olympus 3.3RTV, Japan). After $48 \mathrm{~h}$ of transfection, the supernatant was collected by centrifugation at 4,000 $\mathrm{g}$ for $10 \mathrm{~min}$ at $4^{\circ} \mathrm{C}$, followed by ultrafiltration centrifugation at $4000 \mathrm{~g}$ for $12 \mathrm{~min}$. The concentrated solution was stored at $-80^{\circ} \mathrm{C}$ for future experiments.

Gene transfection. BxPC-3, Panc-1 and MIApaca-2 cells were seeded at a density of $2 \times 10^{5}$ cells/well in 6-well microplates. After growing overnight, the cells were transfected with lentivirus carrying shRNAs against KLF4 or KLF4 cDNA (GeneChem, Shanghai, China). For ZEB1 knockdown, PDAC cells were transfected with siRNA against ZEB1 (GGTAGA TGGTAATGTAATA) using Lipofectamine 3000 (Invitrogen).
Western blot analysis. Western blot analysis was performed as previously described (11). Equal amounts of protein were loaded to sodium dodecyl sulfate polyacrylamide gels. The protein was subsequently transferred onto PVDF membranes. After blocked with 5\% BSA, membranes were incubated with the indicated primary antibodies overnight at $4{ }^{\circ} \mathrm{C}$, followed by treatment with horseradish peroxidase-conjugated secondary antibodies for $1 \mathrm{~h}$ at room temperature. The protein signals were detected using the ECL kit and quantified using an Alpha Imager 2200 (Alpha Innotech Corp., USA).

Northern blot analysis. The total RNA was extracted using TRIzol reagent (Invitrogen). RNA (20 $\mu \mathrm{g})$ was subjected to $15 \%$ urea-polyacrylamide gels, and was then transferred to nylon membrane. The expression of miR-200b, miR-183 and U6 were detected using biotin-labeled oligonucleotide probes (5'-TCATCATTACCAGGCAGTATTA-3', miR-200b; 5'-CAG TGAATTCTACCAGTGCCAT-3', miR-183 and 5'-ATATG GAACGCTTCACGAATT-3', U6). The signals of blots were measured using the Fujifilm LAS-4000 imaging system.

Luciferase reporter assay. Panc-1 and MIApaca-2 cells were co-transfected with increasing concentrations of pcDNA3.1 carrying KLF4 and $0.2 \mu \mathrm{g}$ of luciferase reporter plasmid containing the miR-200b or miR-183 promoter using Lipofectamine 3000 (Roche); the pRL-SV40 plasmid (Promega) was transfected as a normalization control. After 24-h incubation, cell lysates were collected to determine luciferase activity using the Dual-Luciferase assay (Promega) according to the manufacturer's instructions. The primers were used as follows: 5'-ATATGCGGCCGCGTTTTGGCTTCGTTTCTTCT-3' and 5'-GCTTGGTACCGACCCCATCTGTTCTTTGATT-3' for KLF4; 5'-AGAAAGGGTGGGAAGGAGGACA-3' and 5'-GGACTCGCTGGGAAGCTCAGTA-3' for miR-200b; 5'-ATTGTAGTAAGGGAAACTGAGGC-3' and 5'-GCAGAA GTGGGTAAGGTGCT-3' for miR-183. The sequence of ZEB1-3'UTR containing the miR-200b seed regions were amplified using specific primers (forward, 5'-CGAGCTCATT GTTTTATCTTATCAGTATTATC-3'; reverse, 5'-CCGCTCG AGAACTAAAAGAAATAAAATAATACTG-3') SacI and $X h o I$ restriction sites. The PCR product was digested using the restriction enzymes $S a c I$ and XhoI, and was subsequently linked to pHSA-MIR-Report (Ambion). Mutations in the miR-200b seed regions of the ZEB1-3'UTR were generated using the QuikChange Multi-site-directed mutagenesis kit (Stratagene). For miR-183, two targeting sequences locating at position 880-886, 1801-1807 of ZEB1-3'UTR were amplified using forward (5'-CGAGCTCAGTGCCATTTCTCAGTATT TTCAAG-3') and reverse (5'-CCGCTCGAGAGTGCCAT TTCTCAGTATTTTCAAG-3') primers containing SacI and XhoI restriction sites. The corresponding mutagenesis was generated using specific primers (forward, 5'-CGAGCTCACAC GGATTTCTCAGTATTTTCAAG-3'; reverse, 5'-CCGCT CGAGAGACGGATTTCTCAGTATTTTCAAG-3').

Chromatin immunoprecipitation assay (ChIP). ChIP was performed using a ChIP assay kit (Millipore) according to the manufacturer's instructions. In brief, Panc-1 cells were crosslinked with fresh $1 \%$ formaldehyde, followed by incubation in SDS lysis buffer containing $1 \%$ protease inhibitors. 
Table I. Primers for miR-200b.

\begin{tabular}{lll}
\hline Sites & \multicolumn{1}{c}{ Forward primer } & \multicolumn{1}{c}{ Reverse primer } \\
\hline 1 & 5'-TCAGACCCCAGACCAGCA-3' & 5'-CCACCTGGAGACCCCTCT-3' \\
2 & 5'-GTGGCGGGGACCGTTCTGT-3' & 5'-TCTGAGGCAGGGGACAA-3' \\
3 & 5'-CCTTGTCCCCTGCCTCA-3' & 5'-CCTTCCCTCTGGGTGGTC-3' \\
4 & 5'-GCCTTCCTATGGGACCACC-3' & 5'-CGAGTCCAGCAGCCACCAG-3' \\
5 & 5'-GGCAGAGGGCCCGTGTCA-3' & 5'-GGGTTGCATGGGACTCGCT-3' \\
\hline
\end{tabular}

Table II. Primers for miR-183.

\begin{tabular}{lll}
\hline Sites & \multicolumn{1}{c}{ Forward primer } & \multicolumn{1}{c}{ Reverse primer } \\
\hline 1 & 5'-CAAGCTGGATTGTCCTCTGG-3' & 5'-CCTCTTGGCGATGTTACCC-3' \\
2 & 5'-GCTCCCTCCAAGCCACCT-3' & 5'-CCCCAGGAACAAACCGAAT-3' \\
3 & 5'-TGTTCCTGGGGCTCTGTTT-3' & 5'-GGACCATCCATCTCCTTGC-3' \\
4 & 5'-GTCTGGGTGATGTGGAGGG-3' & 5'-AGCGGCTGCTACGGTCT-3' \\
5 & 5'-TAGCAGCCGCTGCTGAGG-3' & 5'-GCCCACGATGCCCGTT-3"' \\
\hline
\end{tabular}

The cell lysates were sonicated to shear crosslinked DNA to 200-1,000 bp in length. Protein/DNA complexes were immunoprecipitated with $3 \mu \mathrm{g}$ anti-ZEB1 antibody or control IgG. The complexes were eluted from the antibodies, and were dissociated with $5 \mathrm{M} \mathrm{NaCl}$. ChIP samples were analyzed with real-time quantitative PCR using primers specific for the promoters of miR-200b and miR-183. The primers are listed in Tables I and II.

Quantitative RT-PCR. Total RNA was extracted using TRIzol reagent (Invitrogen). The expression of miR-200b and miR-183 were analyzed using TaqMan miRNA assays (Applied Biosystems, CA, USA) according to the manufacturer's instructions. U6 snRNA was used as an internal control.

Immunohistochemistry. Immunohistochemistry was performed as previously described (12). Briefly, tumor sections were fixed in formalin, embedded with paraffin, deparaffinized in xylene, and hydrated with ethanol. After antigen retrieval using microwave, the tissue sections were treated with $3 \%$ hydrogen peroxide and blocked with $10 \%$ goat serum. The sections were then stained with anti-KLF4 and anti-ZEB1 antibodies, followed by treatment with a biotinylated secondary antibody. The images were captured using a fluorescence microscope (Leica DM IRE2).

Mouse xenograft models. The orthotopic PDAC nude mouse model was established as previously described by us (12), with a minor modification. Briefly, 4- to 6-week-old BALB/c nude mice of both sexes were obtained from Weitonglihua Animal Center (Beijing, China) and maintained under specific pathogen-free conditions in the animal facility. All procedures were approved by the Institutional Laboratory Animal Care and Use Committee at Shandong Provincial Hospital. Panc-1 cells $\left(1 \times 10^{7}\right)$ in $100 \mu \mathrm{l}$ PBS/Matrigel (1:1, v/v, BD Biosciences,
USA) transfected with control lentivirus or lentivirus carrying KLF4 cDNA were injected into both flanks of the mice. One week after injection, gemcitabine ( $80 \mathrm{mg} / \mathrm{kg}$; q3 days) was administered via intraperitoneal injection for six weeks. The long (L) and short axes (S) of the tumors were measured weekly using Vernier calipers, and the tumor volumes were calculated as follows: $\mathrm{V}=\left(\mathrm{L}_{\mathrm{x}} \mathrm{S}^{2}\right) \times \pi / 6$. The mice were sacrificed $24 \mathrm{~h}$ after the last injection, and the tumors were harvested for subsequent analysis.

Statistical analysis. Statistical analysis was carried out using the SPSS 18.0 software package. All data are presented as the mean \pm standard deviation from at least three independent experiments. Student's t-test was used for comparisons between two groups. One-way ANOVA was used for comparisons among multiple groups. $\mathrm{p}<0.05$ was considered statistically significant.

\section{Results}

Gemcitabine inhibits the expression of KLF4, miR-200b and miR-183 and promotes ZEB1 expression. First, we measured the expression of KLF4 and ZEB1 in BxPC-3, Panc-1 and MIApaca-2 cells. Fig. 1A showed a higher level of KLF4 in BxPC-3 cells than those in Panc- 1 and MIApaca- 2 cells while lower levels of ZEB1 in BxPC-3 cells than those in Panc-1 and MIApaca- 2 cells. Exposure of BxPC-3 cells to gemcitabine caused more apparent decline in cell viability $\left(\mathrm{IC}_{50}=3.942 \mu \mathrm{M}\right)$ compared to Panc-1 $\left(\mathrm{IC}_{50}=9.383 \mu \mathrm{M}\right)$ and MIApaca- 2 cells $\left(\mathrm{IC}_{50}=7.904 \mu \mathrm{M}\right)$ (Fig. 1B). Flow cytometry analysis demonstrated that treatment with $10 \mu \mathrm{M}$ gemcitabine induced the death of $68.34 \%$ of the BxPC-3 cells, $50.45 \%$ of the Panc-1 cells and $51.02 \%$ of the MIApaca-2 cells (Fig. 1C), suggesting that BxPC-3 cells were more sensitive to gemcitabine than Panc-1 and MIApaca-2 cells. Further investigation found 
A

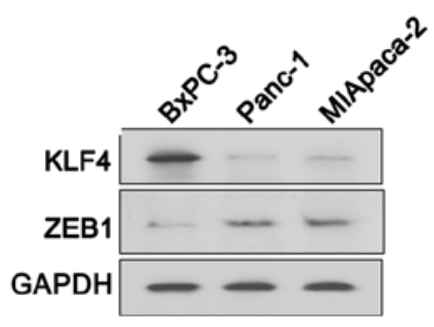

B

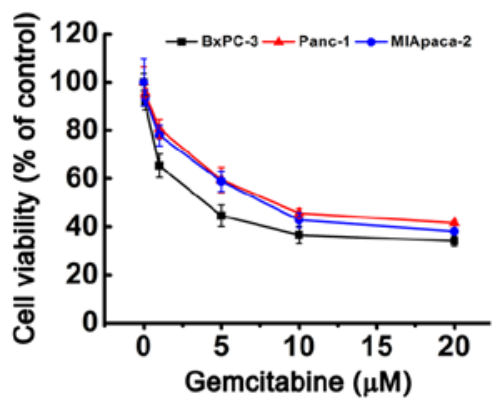

$\mathbf{E}$

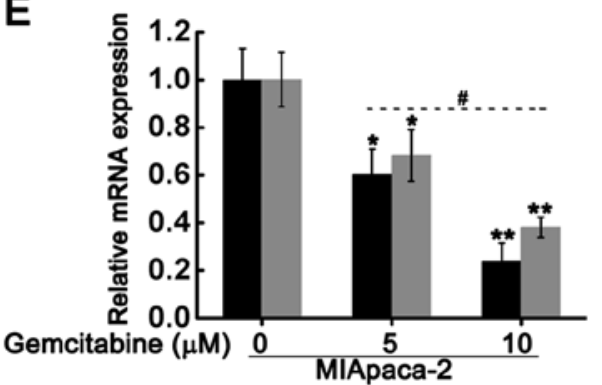

C
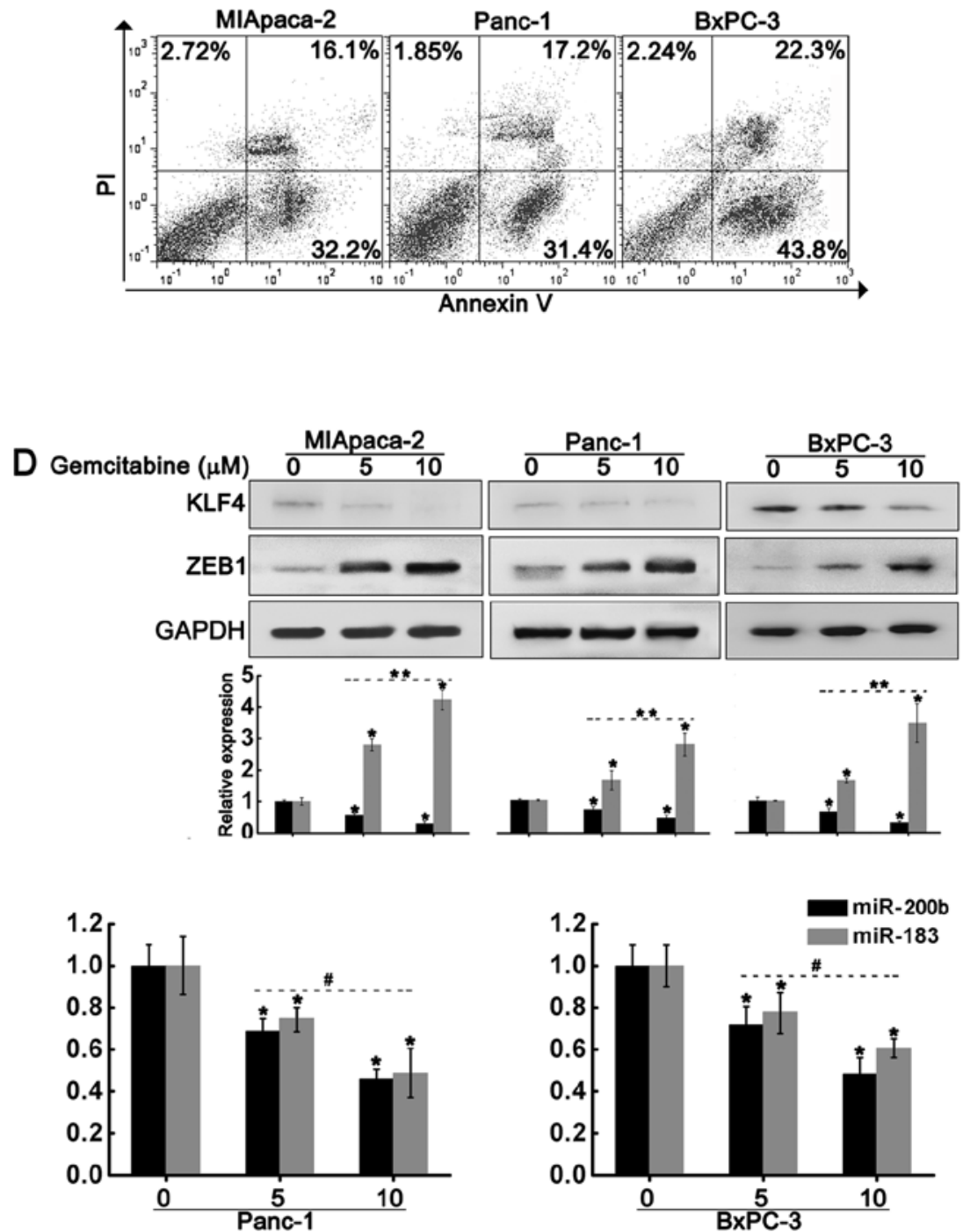

Figure 1. Gemcitabine reduces the expression of KLF4, miR-200b and miR-183, but promoted ZEB1 expression. (A) Western blot analysis was used to analyze the expression of KLF4 and ZEB1 in BxPC-3, Panc-1 and MIApaca-2 cells. *p<0.01. (B) Cell viabilities of BxPC-3, Panc-1 and MIApaca-2 cells exposed to different concentrations of gemcitabine. (C) Flow cytometric analysis of cell apoptosis induced by $10 \mu \mathrm{M}$ gemcitabine. (D) BxPC-3, Panc-1 and MIApaca-2 cells were treated with the indicated concentration of gemcitabine for $72 \mathrm{~h}$. The expression of KLF 4 and ZEB1 were analyzed by western blot analysis. ${ }^{*}<0.01$; ${ }^{* *} \mathrm{p}<0.05$. (E) BxPC-3, Panc-1 and MIApaca-2 cells were treated with the indicated concentration of gemcitabine for $72 \mathrm{~h}$. The expression of miR-200b and miR-183 were analyzed by RT-PCR. ${ }^{*} \mathrm{p}<0.05 ;{ }^{* *} \mathrm{p}<0.01 ;{ }^{\#} \mathrm{p}<0.05$.

that gemcitabine treatment reduced the expression of KLF4, miR-200b and miR-183 in a dose-dependent manner, whereas led to a dose-dependent increase in ZEB1 expression (Fig. 1D and E).

KLF4 attenuates ZEB1 expression and gemcitabine resistance by upregulation of miR-200b and miR-183. Next, we examined the correlation between KLF4 and ZEB1. KLF4 knockdown significantly promoted ZEB1 expression while KLF4 overexpression inhibited ZEB1 expression (Fig. 2A). KLF4 depletion inhibited gemcitabine-induced activation of caspase 3 while KLF4 overexpression promoted the activation of caspase 3 even in the absence of gemcitabine (Fig. 2B), suggesting that KLF4 downregulation conferred gemcitabine resistance of PDAC cells. KLF4 knockdown restrained the expression of miR-200b and miR-183 (Fig. 2C). In contrast,
KLF4 overexpression elevated the levels of miR-200b and miR-183 (Fig. 2C). In KLF4-depleted cells, forced expression of miR-200b and miR-183 repressed the ZEB1 expression again (Fig. 2D). In KLF4-overexpressing cells, forced expression of anti-miR-200b and anti-miR-183 restored the ZEB1 expression (Fig. 2E). Restoration of miR-200b and miR-183 activated caspase 3 in KLF4-depleted cells whereas anti-miR-200b and anti-miR-183 significantly reduced caspase 3 activation in KLF-overexpressing cells (Fig. 2F). Overexpression of miR-200b and miR-183 in KLF-4-depleted cells promoted cell apoptosis in the presence of gemcitabine, but inhibition of miR-200b and miR-183 in KLF-4-overexpressing cells blocked cell apoptosis induced by gemcitabine (Fig. 2G). These results suggested that KLF4 knockdown contributed to ZEB1 expression and gemcitabine resistance by suppressing miR-200b and miR-183. 
A
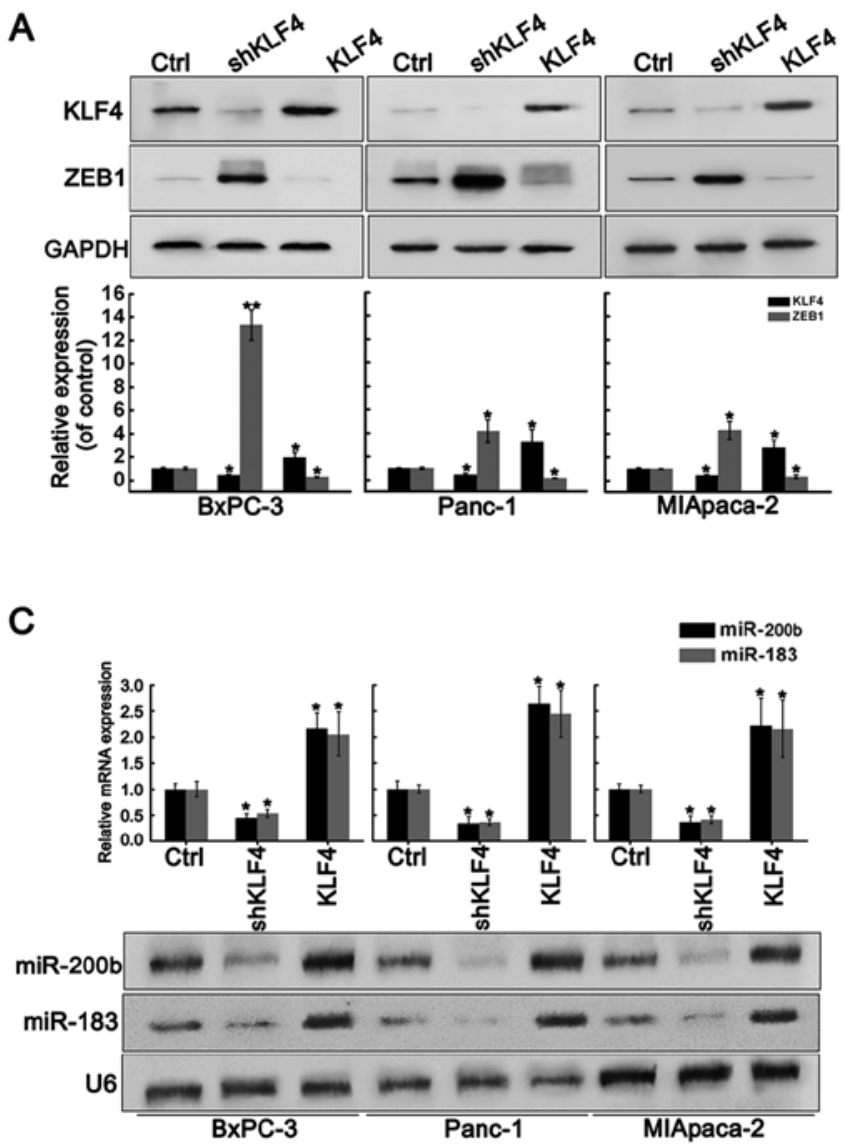

F

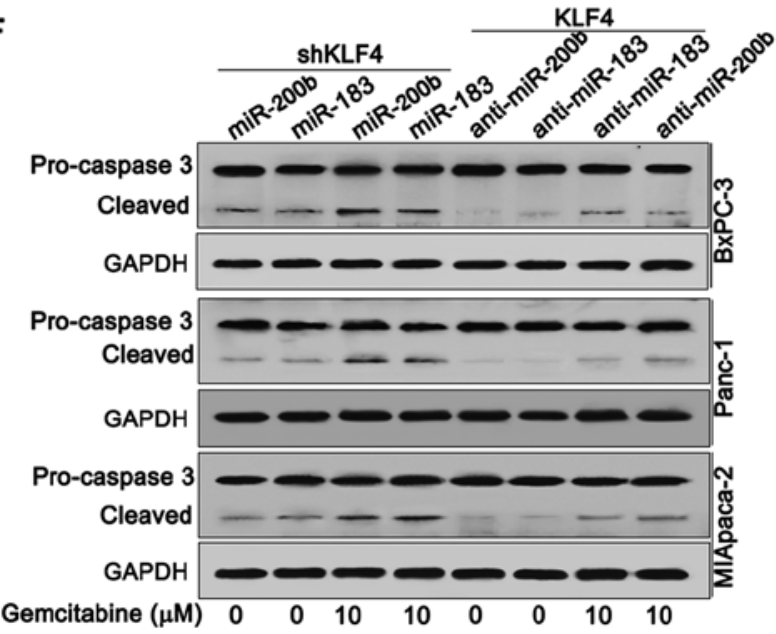

B

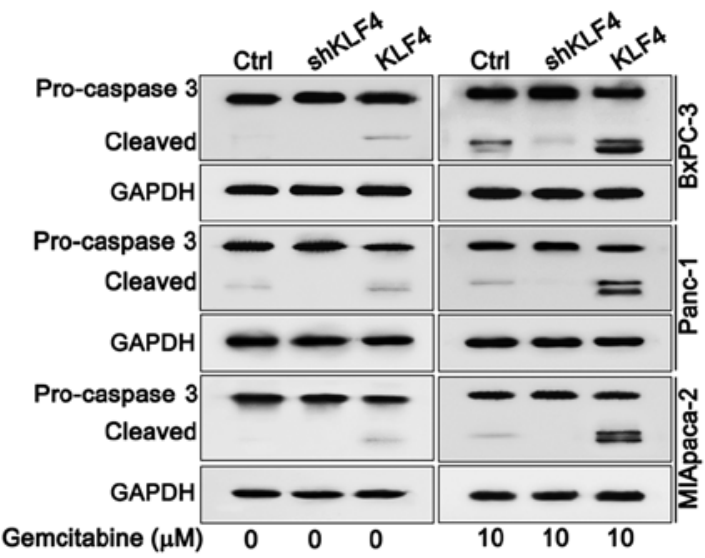

D

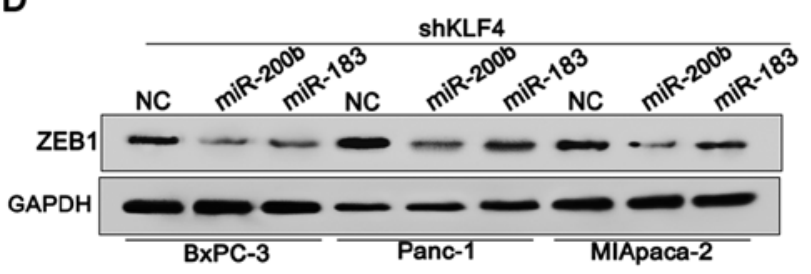

E

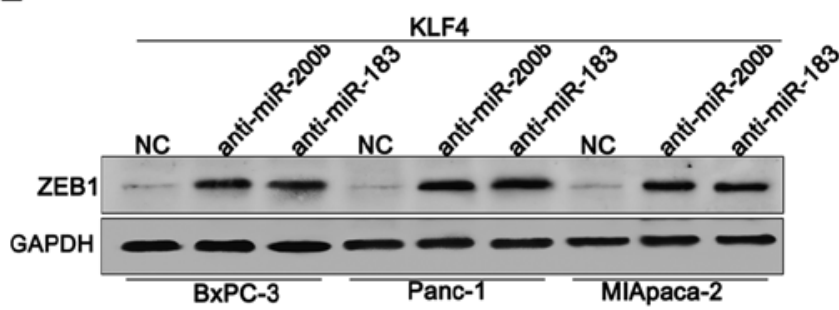

G

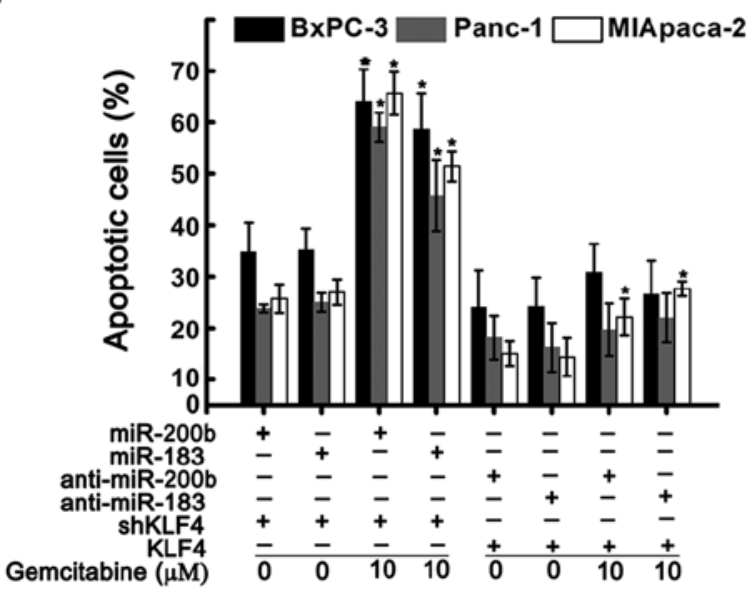

Figure 2. KLF4 attenuates ZEB1 expression and gemcitabine resistance by elevating the levels of miR-200b and miR-183. (A) BxPC-3, Panc-1 and MIApaca-2 cells were transfected with lentivirus carrying shRNAs against KLF4 or KLF4 cDNA. The expression of KLF4 and ZEB1 were analyzed by western blot analysis. ${ }^{*}$ p $<0.01$ vs. control; ${ }^{* *}$ p $<0.001$ vs. control. (B) The KLF4-depleted or KLF4-overexpressing cells were treated with $10 \mu \mathrm{M}$ gemcitabine. The activation of caspase 3 was determined by western blot analysis. (C) BxPC-3, Panc-1 and MIApaca-2 cells were transfected with lentivirus shRNAs against KLF4 or KLF4 cDNA. The expression of miR-200b and miR-183 were analyzed by RT-PCR and northern blot analysis. (D) The KLF4-depleted cells were transfected with miR-200b and miR-183. The expression of ZEB1 was analyzed by western blot analysis. (E) The KLF4-overexpressing cells were transfected with anti-miR-200b and anti-miR-183. The expression of ZEB1 was analyzed by western blot analysis. (F) The KLF4-depleted cells were transfected with miR-200b and miR-183, followed by treatment with $10 \mu \mathrm{M}$ gemcitabine. The KLF4-overexpressing cells were transfected with anti-miR-200b and antimiR-183, followed by treatment with $10 \mu \mathrm{M}$ gemcitabine. The activation of caspase 3 was determined by western blot analysis. Cell viabilities were determined by CCK 8 assay $(\mathrm{G}) .{ }^{*} \mathrm{p}<0.01$

KLF4 positively regulates the expression of $m i R-200 \mathrm{~b}$ and miR-183 in PDAC cells. Since KLF4 is a transcriptional factor that regulates the expression of multiple genes, we hypothesized that KLF4 directly regulated the expression of miR-200b and miR-183. The putative binding sites of KLF4 located on the promoters of miR-200b and miR-183 were 

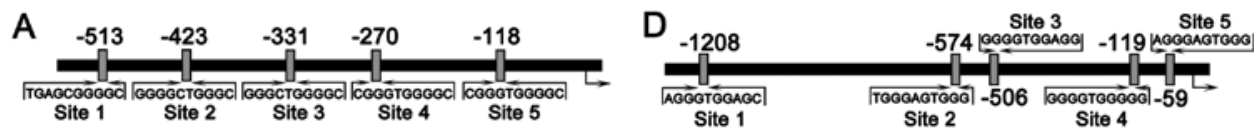

B

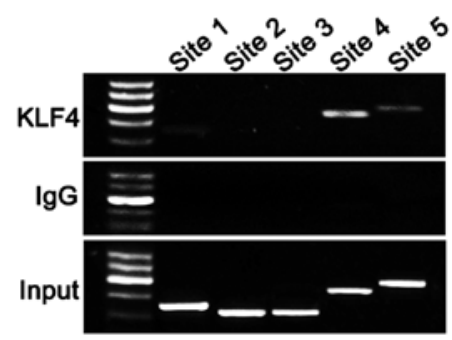

E

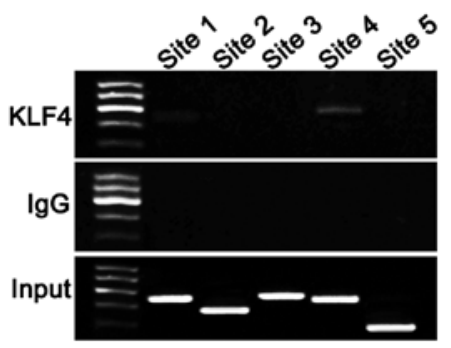

C

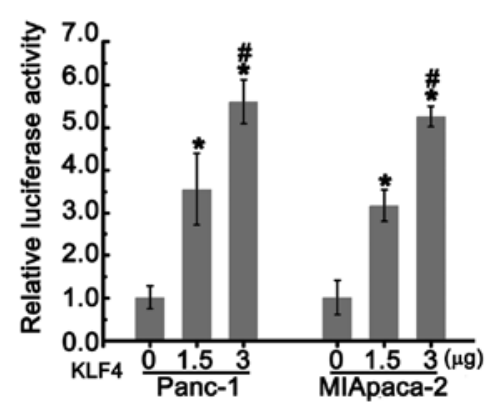

$\mathbf{F}$

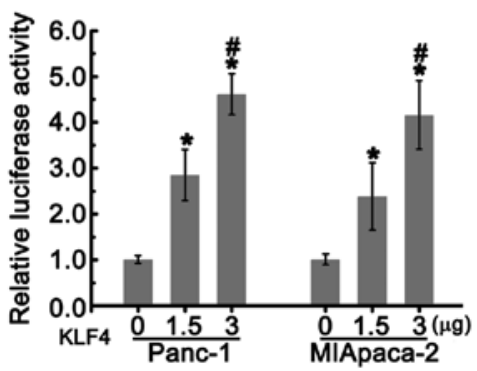

Figure 3. KLF4 directly regulates the expression of miR-200b and miR-183 by binding to their promoters. (A) The putative binding sites of KLF4 in the miR-200b promoter are shown. (B) ChIP assays identified the binding sites of KLF4 in the miR-200b promoter. (C) Panc-1 and MIApaca-2 cells were co-transfected with increasing concentrations of pcDNA3.1 plasmid carrying KLF4 and $0.2 \mu \mathrm{g}$ pGL3-200b-luc using Lipofectamine 3000. After 48-h transfection, luciferase activity was measured. "p $<0.01$ vs. control group; ${ }^{\#} \mathrm{p}<0.05$ vs. the group transfected with $0.1 \mu \mathrm{g}$ pcDNA3.1 carrying KLF4. (D) The putative binding sites of KLF4 in miR-183 promoter are shown. (E) ChIP assays identified the binding sites of KLF4 in miR-183 promoter. (F) Panc-1 and MIApaca-2 cells were co-transfected with increasing concentrations of pcDNA3.1 plasmid carrying KLF4 and 0.2 $\mu \mathrm{g}$ pGL3-200b-luc using Lipofectamine 3000. After 48-h transfection, luciferase activity was measured. " $\mathrm{p}<0.05$ vs. control group; ${ }^{*} \mathrm{p}<0.05$ vs. the group transfected with $0.1 \mu \mathrm{g}$ pcDNA3.1 carrying KLF4.

shown in Fig. 3A and D. Chromatin immunoprecipitation (ChIP) assays revealed that KLF4 could interact with site 4 and 5 in the miR-200b promoter and the site 4 in the miR-183 promoter (Fig. 3B and E). We subsequently constructed the pGL3-200-luc and pGL3-183-luc plasmids by inserting these sequences into the pGL3 luciferase reporter. Panc-1 and MIApaca- 2 cells were co-transfected with $0.2 \mu \mathrm{g}$ pGL3-200-luc or pGL3-183-luc and the indicated concentration of pcDNA3.1 carrying KLF4 together with $100 \mathrm{ng}$ pRL-SV40. As shown in Fig. 3C and F, with the increasing dose, KLF4 substantially enhanced luciferase activities. These data suggested that KLF4 positively regulated the expression of miR-200b and miR-183 by directly binding to their promoters.

miR-200b and miR-183 directly targets ZEB1 in PDAC cells. We further explored whether miR-200b and miR-183 directly targeted the-3'UTR region of ZEB1. Targetscan (http://www. targetscan.org/) was used to predict the recognition sites of miR-200b and miR-183. As shown in Fig. 4A, four putative sites for miR-200b and two putative sites for miR-183 were found. The sequences containing these sites were cloned into the luciferase reporter plasmid to obtain the ZEB1-3'UTRWT-luc plasmid (wild-type), and the sequence containing the mutant sites was cloned to construct ZEB1-3'UTR-MT-luc plasmid (mutant type). Subsequently, the Panc-1 cells were co-transfected with ZEB1-3'UTR-WT or ZEB1-3'UTR-MT and miR-200b or miR-183. Both miR-200b and miR-183 significantly inhibited luciferase activities in the cells carrying ZEB1-3'UTR-WT compared to those carrying negative control (NC) and ZEB1-3'UTR-MT (Fig. 4B). Furthermore, both miR-200b and miR-183 notably reduced mRNA and protein levels of ZEB1 in BxPC-3, Panc-1 and MIApaca-2 cells (Fig. 4C and D). Conversely, both anti-miR-200b and anti-miR-183 elevated mRNA and protein levels of ZEB1 (Fig. 4C and D). These results indicated that miR-200b and miR-183 directly targeted ZEB1.

ZEB1 knockdown contributes to gemcitabine sensitivity of PDAC cells. As shown in Fig. 5A, gemcitabine treatment promoted ZEB1 expression, which was consistent with the results in Fig. 1D.ZEB1 knockdown mildly activated caspase 3. A combination of ZEB1 knockdown and gemcitabine significantly increased cleaved caspase 3, suggesting that ZEB1 knockdown facilitated gemcitabine sensitivity of PDAC cells. Fig. 5B illustrated that gemcitabine treatment induced cell apoptosis in ZEB1-depleted cells. These data indicated that ZEB1 knockdown contributed to gemcitabine sensitivity of PDAC cells.

Negative association between KLF4 and ZEBI regulates gemcitabine resistance of PDAC in vivo. We established xenograft tumors of PDAC to determine the effect of KLF4 overexpression on the ZEB1 expression and gemcitabine resistance of PDAC in vivo. The results showed that KLF4 
A

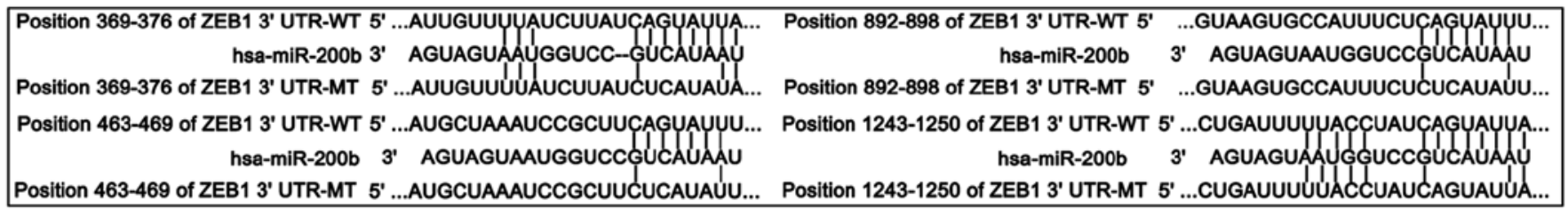

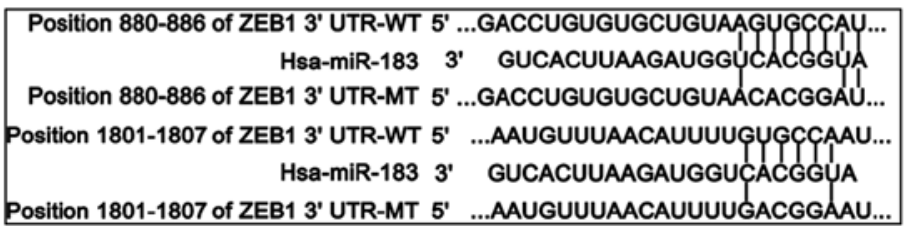

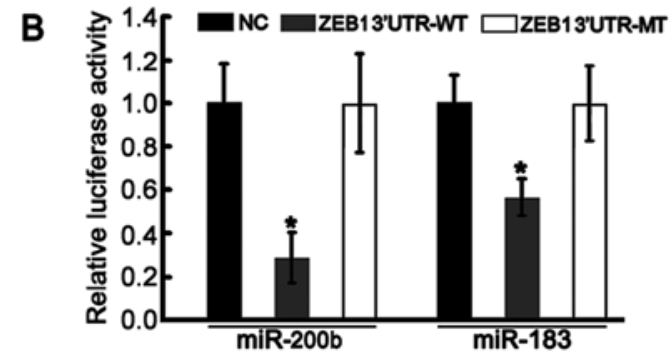

C

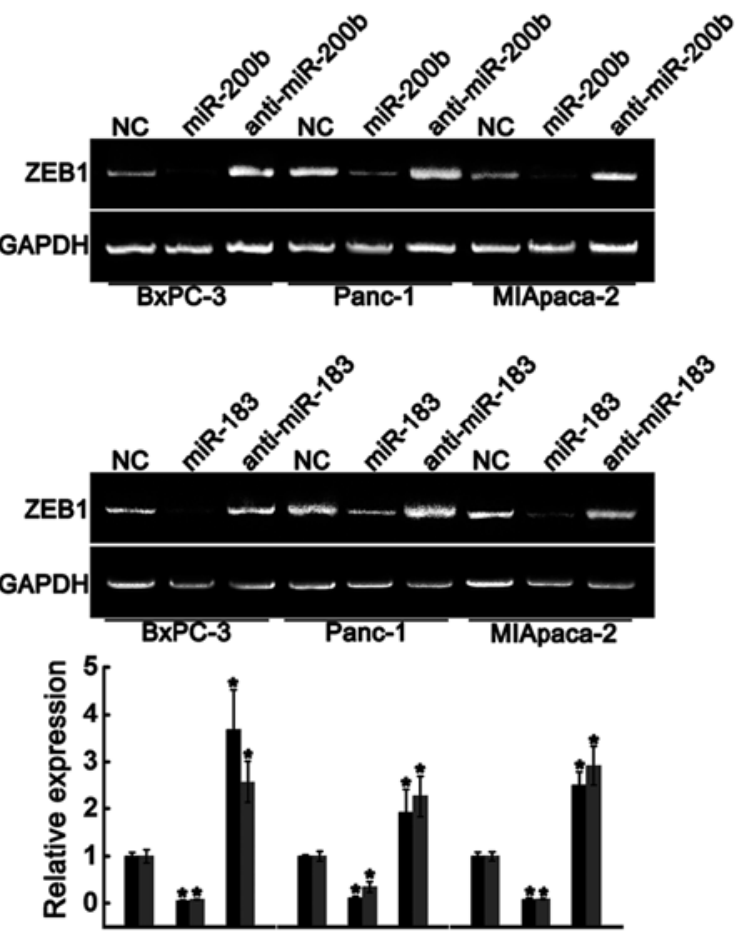

D
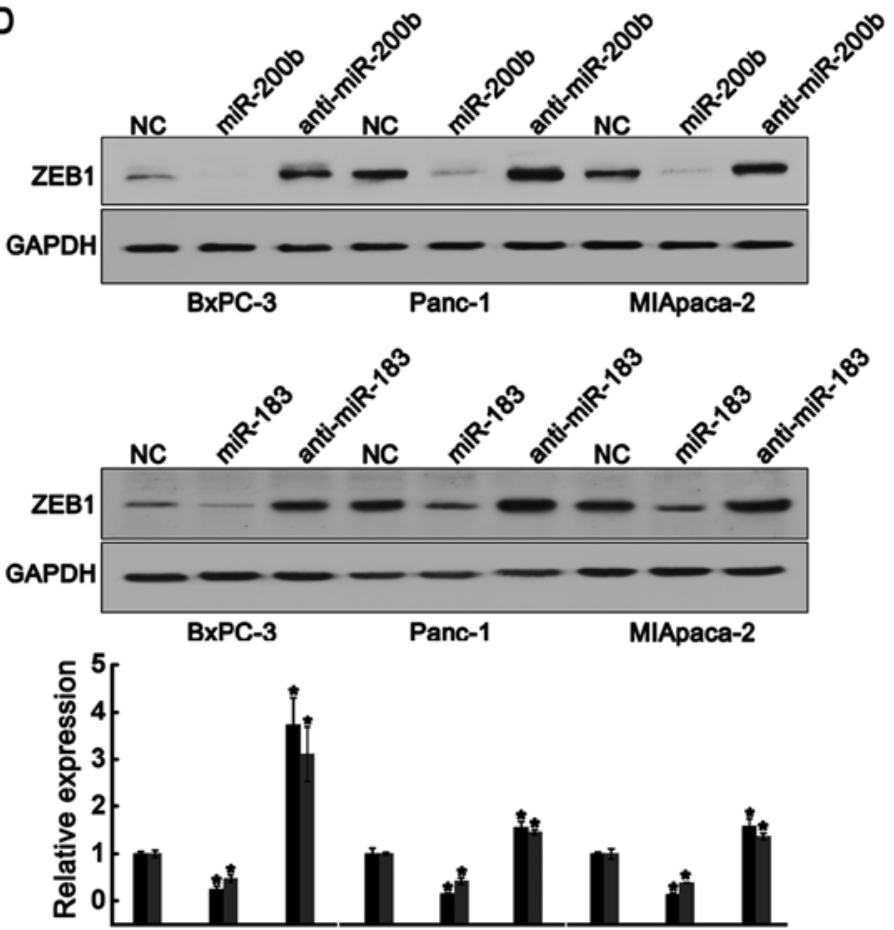

Figure 4. miR-200b and miR-183 directly targets ZEB1 in PDAC cells. (A) Targeting sequences of miR-200b and miR-183 in the-3'UTR region of ZEB1. (B) Panc-1 cells were co-transfected with miR-200b or miR-183 and ZEB1-3'UTR-WT or ZEB1-3'UTR-MT. The relative luciferase activities were determined using the Dual-Luciferase assay. " p<0.01 vs. NC group. (C) BxPC-3, Panc-1 and MIApaca-2 cells were transfected with miR-200b, miR-183, anti-miR-200b or anti-miR-183. The mRNA expression of ZEB1 was determined by RT-PCR. " $\mathrm{p}<0.01$ vs. NC group. The protein expression of ZEB1 was determined by western blot analysis (D). * p $<0.01$ vs. NC group.

overexpression reduced ZEB1 expression, consistent with the data in vitro (Fig. 6A-C). Linear regression analysis showed that KLF4 expression was negatively correlated to ZEB1 expression (Fig. 6D). Compared to the control group, KLF4 overexpression did not significantly affect tumor growth, whereas it enhanced gemcitabine sensitivity (Fig. 6E and F). These results suggested that KLF4 overexpression attenuated gemcitabine resistance of PDAC in vivo by negatively regulating ZEB1 expression.

\section{Discussion}

Chemotherapy resistance has been a major challenge in improving the overall survival of patients with PDAC.
Gemcitabine is a standard clinical chemotherapeutic drug for advanced PDAC, but results in a progression-free survival interval ranging from 0.9 to 4.2 months only (12). Thus, understanding of the mechanism by which chemotherapy resistance occurs contributes to the development of novel therapeutic strategies for overcoming advanced PDAC. KLF4 has been identified as a tumor suppressor in diverse types of cancer (13-15), including PDAC (5). In the pancreas, KLF4 is predominantly expressed in PDAC and regulates the expression of cytokeratin-19, a specific marker for PDAC (16). In this study, we observed a higher level of KLF4 in BxPC-3 cells compared to those in Panc-1 and MIApaca- 2 cells. Intriguingly, BxPC-3 cells were more sensitive to gemcitabine than Panc-1 and MIApaca-2. Fig. 1D illustrated that gemcitabine treatment 


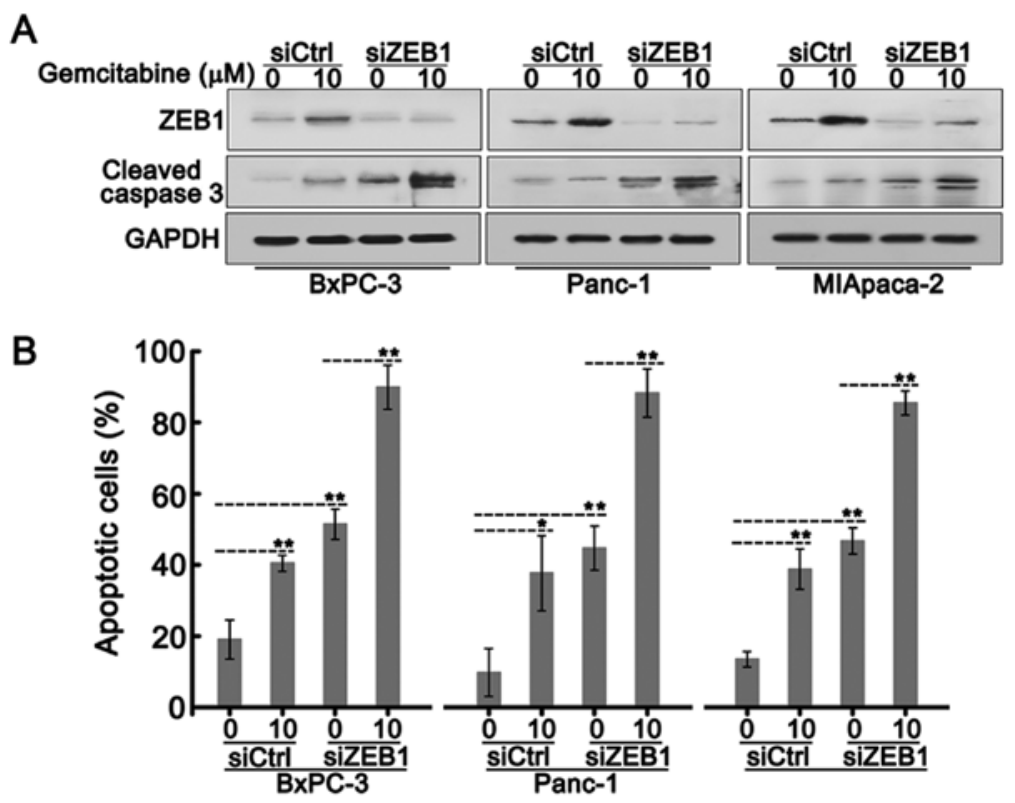

Figure 5. ZEB1 knockdown attenuates gemcitabine resistance in PDAC cells. BxPC-3, Panc-1 and MIApaca-2 cells were transfected with siRNA against ZEB1 for $48 \mathrm{~h}$, followed by treatment with $10 \mu \mathrm{M}$ gemcitabine. (A) The expression of ZEB1 and cleaved caspase 3 were analyzed by western blot analysis. (B) Cell viabilities were determined by CCK 8 assay. ${ }^{*} \mathrm{p}<0.05 ;{ }^{* *} \mathrm{p}<0.01$
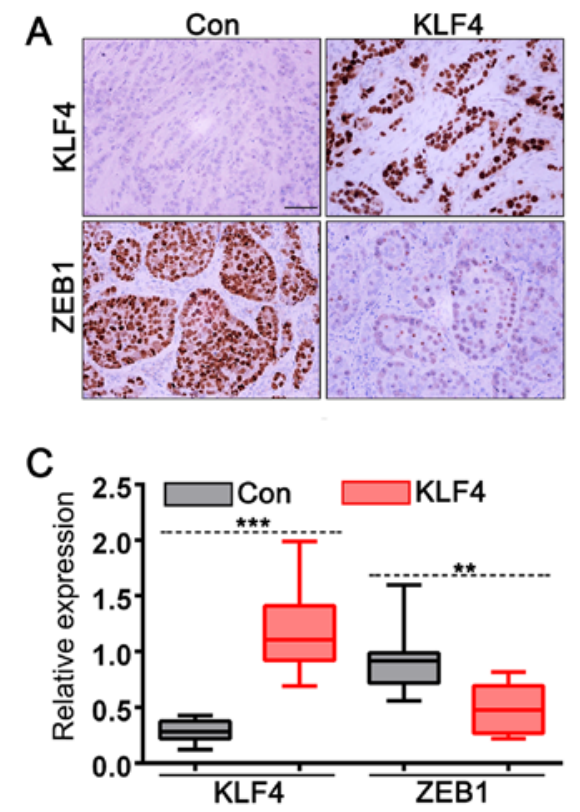

$\mathrm{E}$

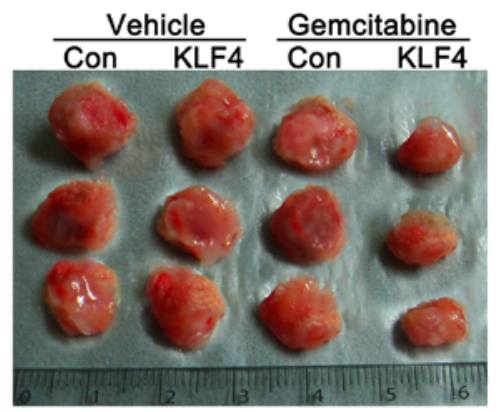

B

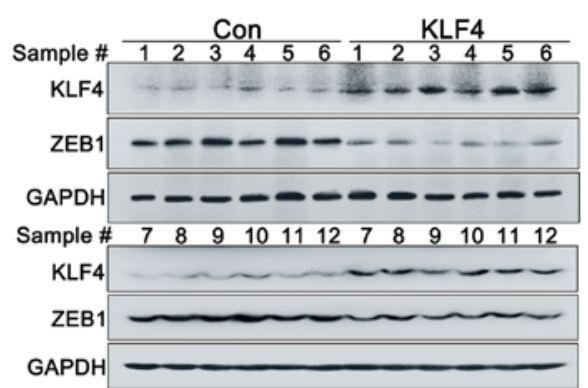

D

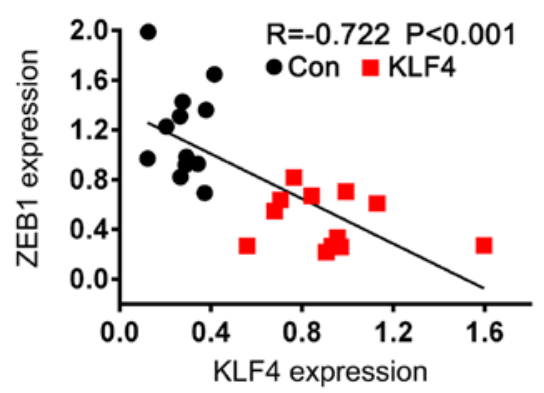

$\mathbf{F}$

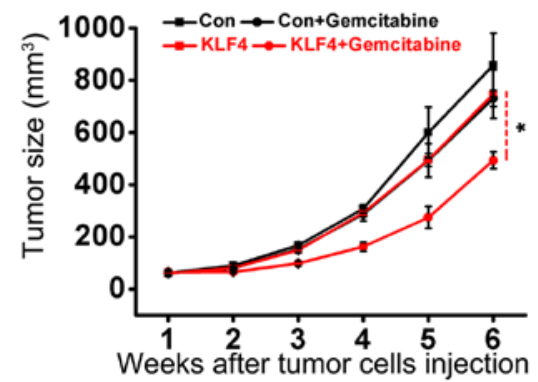

Figure 6. KLF4 reduces gemcitabine resistance of PDAC in vivo by negatively regulating ZEB1 expression. (A) Representative IHC staining for KLF4 and ZEB1 in tumor tissues from control mice or KLF4 expressing mice. (B) Western blot analysis of KLF4 and ZEB1 in tumor tissues from control mice or KLF4 expressing mice. (C) The relative expression of KLF4 and ZEB1 in tumor tissues from control mice or KLF4 expressing mice. (D) Linear regression analysis of KLF4 and ZEB1 expression was performed using GraphPad Prism 6.0 software. (E) Xenograft tumors from control mice and KLF4 expressing mice are shown. (F) Growth curves of tumors from control mice or KLF4 expressing mice after gemcitabine treatment. ${ }^{*} \mathrm{p}<0.05 ;{ }^{* * *} \mathrm{p}<0.01 ;{ }^{* * * *} \mathrm{p}<0.001$. 


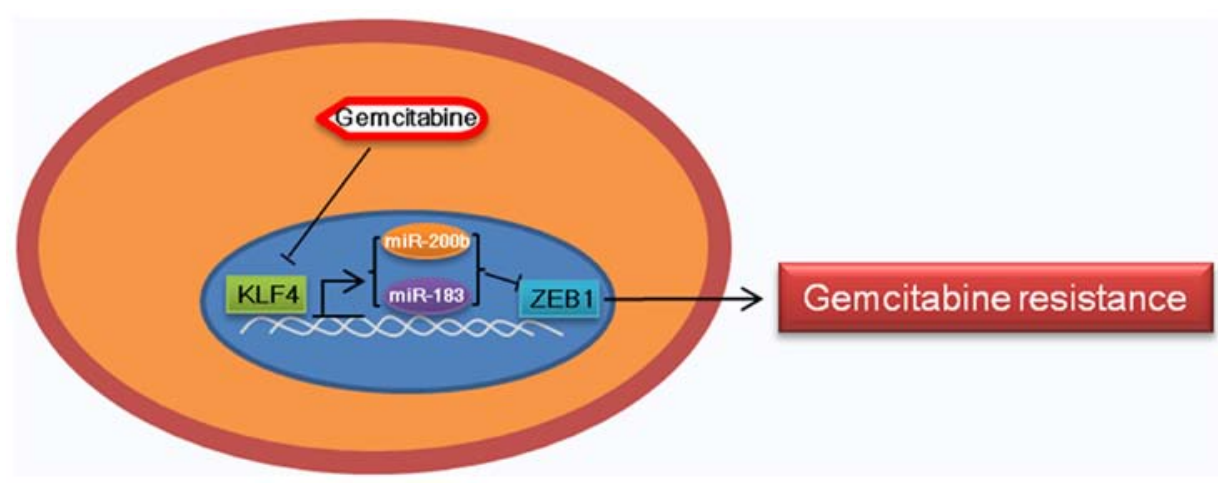

Figure 7. Schematic diagram illustrating crosstalk between KLF4 and ZEB1 in regulating gemcitabine resistance in pancreatic ductal adenocarcinoma.

led to a dose-dependent decrease in the expression of KLF4 in BxPC-3, Panc-1 and MIApaca-2 cells. These results suggested that downregulation of KLF4 might contribute to gemcitabine resistance of PDAC. A previous study demonstrated that $82.4 \%$ of patients with PDAC lack KLF4 expression, which indicates the implication of KLF4 loss in PDAC progression (17). Our results showed that KLF4 knockdown enhanced gemcitabine resistance of PDAC cells while KLF4 overexpression reduced gemcitabine resistance. These results confirmed that the low level of KLF4 promoted gemcitabine resistance of PDAC cells.

How does KLF4 regulate gemcitabine resistance of PDAC cells? Our results showed that gemcitabine treatment simultaneously triggered the downregulation of KLF4, miR-200b and miR-183 in PDAC cells. KLF4 silencing inhibited the expression of miR-200b and miR-183. In contrast, KLF4 overexpression promoted the expression of miR-200b and miR-183. ChIP assays revealed that KLF4 positively regulated the expression of miR-200b and miR-183 by directly binding to their promoters. These results suggested that lower expression of KLF4 conferred PDAC cells with greater gemcitabine resistance by upregulation of miR-200b and miR-183. Consistently, the expression of miR-200b has been found to be significantly downregulated in gemcitabineresistant cells (18). The expression of miR-183 is related to expression of miR-200 family members (19). miR-183 and miR-200 family members cooperate to affect stemness properties in PDAC cells by suppressing Bmil (19), function as EMT inhibitors and accelerate epithelial differentiation by targeting the $\mathrm{Wnt} / \beta$-catenin signaling pathway $(20,21)$. In this study, overexpression of miR-183 and miR-200b reduced the expression of ZEB1 at both mRNA and protein levels in PDAC cells, even in KLF4-depleted cells that ZEB1 was strongly increased. Conversely, anti-miR-200b and anti-miR-183 increased the mRNA and protein levels of ZEB1, even in KLF4-overexpressing cells in which ZEB1 was significantly inhibited. Combined with the data from luciferase reporter assays, we confirmed that miR-200b and miR-183 targeted ZEB1 in PDAC cells. Congruously, ZEB1 has been found to be direct target of miR-200b in hepatocellular carcinoma, osteosarcoma and non-small cell lung cancer (22-24). Although it has been confirmed that ZEB1 represses the transcription of miR-183 in lung cancer $(25,26)$, it is unclear whether miR-183 directly targets ZEB1. Our results indicated that KLF4 knockdown enhanced gemcitabine resistance by suppressing the miR-200b/miR-183/ZEB1 signaling pathway.
ZEB1 is an EMT-activator that is correlated to tumor metastasis, drug resistance and poor prognosis in different tumor types (27). A series of studies have suggested that EMT promotes early-stage dissemination, invasion and metastasis of PDAC $(28,29)$. A recent study demonstrates that EMT is dispensable for metastasis but increases chemoresistance in pancreatic cancer (30). Our results also demonstrated that ZEB1 knockdown promoted gemcitabine sensitivity of PDAC cells.

In conclusion, we demonstrated that gemcitabine inhibited the expression of KLF4, blocking KLF4-mediated downstream signals. Further investigation confirmed that KLF4 induced the expression of miR-200b and miR-183 by directly binding to the promoters of miR-200b and miR-183. Elevated miR-200b and miR-183 subsequently inhibited ZEB1 expression by directly targeting the-3'UTR of ZEB1. Inhibition of ZEB1 attenuated gemcitabine resistance. In addition, KLF4 overexpression enhanced gemcitabine sensitivity of PDAC in vivo by inhibiting ZEB1 expression. Taken together, our results suggested that novel crosstalk between KLF4 and ZEB1 regulates gemcitabine resistance in PDAC cells (Fig. 7).

\section{Acknowledgements}

This study was supported by the National Natural Science Foundation of China (grant nos. 81572272 and 81201778), Shandong Provincial Natural Science Foundation (grant no. ZR2013HQ026) and the Science and Technology Development Plan Project of Shandong Province (grant nos. 2013G0021810 and 2016 GSF201127).

\section{References}

1. Mohammed A, Janakiram NB, Brewer M, Ritchie RL, Marya A, Lightfoot S, Steele VE and Rao CV: Antidiabetic drug metformin prevents progression of pancreatic cancer by targeting in part cancer stem cells and mTOR signaling. Transl Oncol 6: 649-659, 2013.

2. Olive KP, Jacobetz MA, Davidson CJ, Gopinathan A, McIntyre D, Honess D, Madhu B, Goldgraben MA, Caldwell ME, Allard D, et al: Inhibition of Hedgehog signaling enhances delivery of chemotherapy in a mouse model of pancreatic cancer. Science 324: 1457-1461, 2009.

3. Tetreault MP, Yang Y and Katz JP: Krüppel-like factors in cancer. Nat Rev Cancer 13: 701-713, 2013.

4. Wei D, Wang L, Kanai M, Jia Z, Le X, Li Q, Wang H and Xie K: KLF4 $\alpha$ up-regulation promotes cell cycle progression and reduces survival time of patients with pancreatic cancer. Gastroenterology 139: 2135-2145, 2010. 
5. Yan Y, Li Z, Kong X, Jia Z, Zuo X, Gagea M, Huang S, Wei D and Xie K: KLF4-Mediated suppression of CD44 signaling negatively impacts pancreatic cancer stemness and metastasis. Cancer Res 76: 2419-2431, 2016.

6. Rowland BD, Bernards R and Peeper DS: The KLF4 tumour suppressor is a transcriptional repressor of p53 that acts as a context-dependent oncogene. Nat Cell Biol 7: 1074-1082, 2005.

7. Meidhof S, Brabletz S, Lehmann W, Preca BT, Mock K, Ruh M, Schüler J, Berthold M, Weber A, Burk U, et al: ZEB1-associated drug resistance in cancer cells is reversed by the class I HDAC inhibitor mocetinostat. EMBO Mol Med 7: 831-847, 2015.

8. Truesdell P, Ahn J, Chander H, Meens J, Watt K, Yang X and Craig AW: CIP4 promotes lung adenocarcinoma metastasis and is associated with poor prognosis. Oncogene 34: 3527-3535, 2015.

9. Xiong H, Hong J, Du W, Lin YW, Ren LL, Wang YC, Su WY, Wang JL, Cui Y, Wang ZH, et al: Roles of STAT3 and ZEB1 proteins in E-cadherin down-regulation and human colorectal cancer epithelial-mesenchymal transition. J Biol Chem 287: 5819-5832, 2012

10. Rhodes LV, Tate CR, Segar HC, Burks HE, Phamduy TB, Hoang V, Elliott S, Gilliam D, Pounder FN, Anbalagan M, et al: Suppression of triple-negative breast cancer metastasis by pan-DAC inhibitor panobinostat via inhibition of ZEB family of EMT master regulators. Breast Cancer Res Treat 145: 593-604, 2014.

11. Ma J, Fu G, Wu J, Han S, Zhang L, Yang M, Yu Y, Zhang M, Lin Y and Wang Y: 4-cholesten-3-one suppresses lung adenocarcinoma metastasis by regulating translocation of HMGB1, HIF1 $\alpha$ and Caveolin-1. Cell Death Dis 7: e2372, 2016.

12. Xian G, Zhao J, Qin C, Zhang Z, Lin Y and Su Z: Simvastatin attenuates macrophage-mediated gemcitabine resistance of pancreatic ductal adenocarcinoma by regulating the TGF-ß1/Gfi-1 axis. Cancer Lett 385: 65-74, 2017.

13. Wang Z, Li Y, Kong D, Banerjee S, Ahmad A, Azmi AS, Ali S, Abbruzzese JL, Gallick GE and Sarkar FH: Acquisition of epithelial-mesenchymal transition phenotype of gemcitabineresistant pancreatic cancer cells is linked with activation of the notch signaling pathway. Cancer Res 69: 2400-2407, 2009.

14. Yu T, Chen X, Lin T, Liu J, Li M, Zhang W, Xu X, Zhao W, Liu M, Napier DL, et al: KLF4 deletion alters gastric cell lineage and induces MUC2 expression. Cell Death Dis 7: e2255, 2016.

15. Shi M, Cui J, Du J, Wei D, Jia Z, Zhang J, Zhu Z, Gao Y and Xie K: A novel KLF4/LDHA signaling pathway regulates aerobic glycolysis in and progression of pancreatic cancer. Clin Cancer Res 20: 4370-4380, 2014.

16. Li H, Wang J, Xiao W, Xia D, Lang B, Yu G, Guo X, Guan W, Wang Z, Hu Z, et al: Epigenetic alterations of Krüppel-like factor 4 and its tumor suppressor function in renal cell carcinoma. Carcinogenesis 34: 2262-2270, 2013.

17. Wei D, Wang L, Yan Y, Jia Z, Gagea M, Li Z, Zuo X, Kong X, Huang S and Xie K: KLF4 is essential for induction of cellular identity change and acinar-to-ductal reprogramming during early pancreatic carcinogenesis. Cancer Cell 29: 324-338, 2016.

18. Funel N, Morelli M, Giovannetti E, Del Chiaro M, Pollina LE, Mosca F, Boggi U, Cavazzana A and Campani D: Loss of heterozygosity status of D9S105 marker is associated with downregulation of Kruppel-like factor 4 expression in pancreatic ductal adenocarcinoma and pancreatic intraepithelial lesions. Pancreatology 11: 30-42, 2011.
19. Li Y, VandenBoom TG II, Kong D, Wang Z, Ali S, Philip PA and Sarkar FH: Up-regulation of miR-200 and let-7 by natural agents leads to the reversal of epithelial-to-mesenchymal transition in gemcitabine-resistant pancreatic cancer cells. Cancer Res 69: 6704-6712, 2009.

20. Wellner U, Schubert J, Burk UC, Schmalhofer O, Zhu F, Sonntag A, Waldvogel B, Vannier C, Darling D, zur Hausen A, et al: The EMT-activator ZEB1 promotes tumorigenicity by repressing stemness-inhibiting microRNAs. Nat Cell Biol 11: 1487-1495, 2009.

21. Xia H, Cheung WK, Sze J, Lu G, Jiang S, Yao H, Bian XW, Poon WS, Kung HF and Lin MC: miR-200a regulates epithelialmesenchymal to stem-like transition via ZEB2 and beta-catenin signaling. J Biol Chem 285: 36995-37004, 2010.

22. Chen C, Xiang H, Peng YL, Peng J and Jiang SW: Mature miR-183, negatively regulated by transcription factor GATA3, promotes $3 \mathrm{~T} 3-\mathrm{L} 1$ adipogenesis through inhibition of the canonical Wnt/ $\beta$-catenin signaling pathway by targeting LRP6. Cell Signal 26: 1155-1165, 2014.

23. Li Q, Song W, Wang W, Yao S, Tian C, Cai X and Wang L: Suppression of epithelial-mesenchymal transition in hepatocellular carcinoma cells by Krüppel-like factor 4. Oncotarget 7: 29749-29760, 2016.

24. Li Y, Zeng C, Tu M, Jiang W, Dai Z, Hu Y, Deng Z and Xiao W: MicroRNA-200b acts as a tumor suppressor in osteosarcoma via targeting ZEB1. Onco Targets Ther 9: 3101-3111, 2016.

25. Nishijima N, Seike M, Soeno C, Chiba M, Miyanaga A, Noro R, Sugano T, Matsumoto M,Kubota K and Gemma A: miR-200/ZEB axis regulates sensitivity to nintedanib in non-small cell lung cancer cells. Int J Oncol 48: 937-944, 2016.

26. Yang Y, Ahn YH, Chen Y, Tan X, Guo L, Gibbons DL, Ungewiss C, Peng DH, Liu X, Lin SH, et al: ZEB1 sensitizes lung adenocarcinoma to metastasis suppression by PI3K antagonism. J Clin Invest 124: 2696-2708, 2014.

27. Kundu ST, Byers LA, Peng DH, Roybal JD, Diao L, Wang J, Tong P, Creighton CJ and Gibbons DL: The miR-200 family and the miR-183 96 182 cluster target Foxf2 to inhibit invasion and metastasis in lung cancers. Oncogene 35: 173-186, 2016.

28. Lehmann W, Mossmann D, Kleemann J, Mock K, Meisinger C, Brummer T, Herr R, Brabletz S, Stemmler MP and Brabletz T: ZEB1 turns into a transcriptional activator by interacting with YAP1 in aggressive cancer types. Nat Commun 7: 10498, 2016.

29. Galván JA, Zlobec I, Wartenberg M, Lugli A, Gloor B, Perren A and Karamitopoulou E: Expression of E-cadherin repressors SNAIL, ZEB1 and ZEB2 by tumour and stromal cells influences tumour-budding phenotype and suggests heterogeneity of stromal cells in pancreatic cancer. Br J Cancer 112: 1944-1950, 2015.

30. Zheng X, Carstens JL, Kim J, Scheible M, Kaye J, Sugimoto H, Wu CC, LeBleu VS and Kalluri R: Epithelial-to-mesenchymal transition is dispensable for metastasis but induces chemoresistance in pancreatic cancer. Nature 527: 525-530, 2015. 